\title{
Análisis del sistema de operaciones en empresas del sector cerámico español
}

\author{
B. SEGURA' ${ }^{1}$ E. VALLADA ${ }^{2}$, C. MAROTO ${ }^{2}$, R. RUIZ ${ }^{2}$ \\ 'Departamento de Economía y Ciencias Sociales. Universidad Politécnica de Valencia \\ ${ }^{2}$ Departamento de Estadística e Investigación Operativa Aplicadas y Calidad. Universidad Politécnica de Valencia
}

\begin{abstract}
La progresiva desaceleración de la actividad económica a partir de 2001 enfrenta al sector azulejero a retos nuevos en el área de producción. La capacidad productiva y la tecnología están disponibles, fruto de las inversiones en años precedentes y se ha alcanzado una posición ventajosa en el mercado, pero mantener esa posición frente a competidores, tanto tradicionales como emergentes, probablemente requiera un cambio de enfoque en el área de operaciones y sus relaciones con las restantes áreas empresariales. La optimización de todos los recursos disponibles va a necesitar inversiones en procesos y organización más que en capacidad y tecnología. En este trabajo presentamos los resultados de una encuesta sobre el comportamiento de las empresas del sector y las prioridades que declaran para alcanzar los objetivos en las diferentes áreas empresariales, como base para el análisis de su capacidad para dar respuesta a los nuevos retos a los que se enfrentan.
\end{abstract}

Palabras clave: Estrategia empresarial, consenso estratégico, grupos estratégicos

\section{Operations strategy in spanish tile industry firms}

Due to the progressive deceleration of economic activity since 2001, the ceramic tile sector faces new challenges in operations management. Both the capacity and the technology are available, thanks to investments in preceding years, and an advantageous position in the market has been achieved. However, to maintain that position as regards its competitors, traditional as well as emerging ones, a change of focus in operations management and its relationship with business strategy will probably have to be adopted. Resource optimisation needs more investment in process and organization than in capacity and technology. In this study we present the results of a survey on the behaviour of the sector's firms and the priorities they have to reach the objectives, as a basis for the analysis of their capacity to rise to the new challenges with which they are confronted.

Keywords: company strategy, strategy consensus, strategy groups

\section{INTRODUCCIÓN}

El sector productivo de fabricación de pavimentos y revestimientos cerámicos ha experimentado un importante desarrollo en toda la actividad empresarial, que se ha traducido, por una parte, en el crecimiento del volumen de producción, que prácticamente se ha duplicado en los últimos 10 años, y, por otra parte, en la consolidación de su posición en los mercados internacionales con una sólida imagen de calidad.

El crecimiento de la demanda interna de materiales cerámicos, arrastrada por el auge del sector de la construcción, y la competitividad en costes y calidad, que ha permitido desplazar a competidores de otros países, fundamentalmente italianos, unido a unas condiciones favorables del entorno económico global han sido consideradas las principales causas de este desarrollo sectorial (1). La continua expansión de la demanda en cantidad y calidad centraba los problemas organizacionales en capacidad productiva y obtención de productos de calidad. Las inversiones realizadas en la última década, tanto en capacidad como en materiales, diseño y tecnología de producción han situado al sector en una buena posición frente a otros competidores europeos.

La progresiva desaceleración de la actividad económica a partir del año 2001 enfrenta al sector a otros retos en el área de operaciones: la capacidad productiva y la tecnología están disponibles y se ha alcanzado una posición ventajosa en el mercado, debemos preguntarnos por el grado en el que la organización de las empresas del sector es capaz de aprovechar y servir de soporte a las ventajas competitivas conseguidas. Mantener esa posi- ción frente a competidores tanto tradicionales como emergentes probablemente requiera un cambio de enfoque en el área de operaciones y sus relaciones con las restantes áreas empresariales. La optimización de todos los recursos disponibles requiere inversiones en procesos y organización más que en capacidad y tecnología (2). La gestión eficiente de los procesos de producción y una cultura organizacional que establezca un consenso estratégico entre las áreas funcionales permitiría al conjunto del sector mantener su actual posición. Como afirman Lingle y Schiemann, (3) "las organizaciones eficientes son entidades integradas en las que las diferentes unidades, funciones y niveles dan soporte a la estrategia de la empresa y se dan soporte entre sí".

En este trabajo presentamos los resultados de una encuesta sobre el comportamiento de las empresas del sector como base para el análisis de su capacidad para dar respuesta a los retos a los que se enfrentan en la situación actual.

\section{PANORÁMICA DEL SECTOR}

En la actualidad unas 265 empresas se dedican a la actividad productiva de fabricación de pavimentos y revestimientos cerámicos en España; en su inmensa mayoría son pequeñas y medianas empresas y, como es de sobra conocido, concentradas en el ámbito territorial de la provincia de Castellón, que tiene censadas 205 empresas, que obtienen un volumen de producción superior al $94 \%$ del total nacional. Esta fuerte concentración de empresas en la provincia de Castellón ha permitido la creación de 
una red de actividades de producción y servicios auxiliares, entre fabricantes, proveedores, asesores, distribuidores, instituciones financieras especializadas, instituciones públicas de soporte a la $\mathrm{I}+\mathrm{D}+\mathrm{I}$ y a la promoción, etc. que ha facilitado el desarrollo del sector, y probablemente ha tenido más importancia para el mismo que las causas externas mencionadas en la introducción. Esta concentración ha sido considerada como un conglomerado o "cluster" en el sentido establecido en la literatura sobre estrategia empresarial (4), (5) y como tal ha sido objeto de numerosos análisis en los años recientes (6), (7) y (8). En efecto, se ha creado un entorno favorable en el que las empresas pequeñas y medianas han podido desarrollar sus capacidades para que tanto los factores básicos de producción (mano de obra y su coste, las materias primas y los recursos naturales existentes, el capital...) como los factores especializados (el conocimiento científico, el know-how, mano de obra cualificada, recursos financieros adaptados a las necesidades del sector, infraestructuras, etc...) contribuyan al éxito de las empresas y desarrollo del conjunto del sector.

En la literatura sobre la estrategia empresarial se considera que son los factores especializados los que representan ventajas competitivas específicas, en cuanto son la materialización de las capacidades de la empresa, frente a los recursos, representados por los factores básicos mencionados, de carácter más genérico, y por lo tanto, disponibles para cualquier otra empresa (9). No obstante, en el sector cerámico de Castellón se dan otras condiciones adicionales en los factores que han favorecido la consecución de ventajas competitivas. La existencia de recursos naturales (arcilla roja de calidad), y sobre todo, la disponibilidad de mano de obra con formación básica ó especializada, con amplia experiencia en el sector, que se sustenta y potencia con un conjunto de centros docentes que facilitan una formación específica, tanto a nivel Universitario, como de Formación Profesional, sin olvidar la formación continua y ocupación focalizada. Paralelamente se dispone de un conjunto de Instituciones de Investigación, Formación y Representación que atienden al colectivo empresarial, tanto a nivel tecnológico como de diseño y calidad del producto cerámico (10). La actividad de estas instituciones se complementa con una serie de instrumentos bien de promoción, como la feria internacional anual CEVISAMA, bien de divulgación, como el congreso bianual sobre la calidad del azulejo QUALICER o las publicaciones que realizan las instituciones. En este ambiente se dan las condiciones necesarias para facilitar la localización en su entorno a empresas proveedoras, como las de obtención de esmaltes, fritas, colores cerámicos y polvo atomizado. Esta proximidad permite un intercambio de información fluido entre sectores íntimamente relacionados, facilitando la adaptación a los cambios y a las innovaciones en un corto espacio de tiempo. Los programas de actuación conjunta en materia de I+D+I y Diseño entre las empresas de esmaltes y las azulejeras es una muestra de este proceso de intercambio. Paralelamente se ha consolidado la implantación de empresas dedicadas a la fabricación, mantenimiento y, en algunos casos, distribución de bienes de equipo específicos para el proceso productivo desarrollado en el sector. Aunque la tecnología de estos equipos es mayoritariamente de origen italiano, existe una buena interconexión entre sectores. La operación a nivel internacional de unos y otros proveedores ha servido de cauce para la transmisión de tecnología e información. La aglomeración industrial favorece el intercambio de bienes intangibles (información y cooperación). Entre los agentes económicos que operan en el lugar, se genera una cultura empresarial predominante que favorece la adopción de decisiones similares en los sistemas organizativos y se facilitan los acuerdos de colabo- ración para la fabricación en común en algunas fases del proceso productivo. Esta colaboración estimula la creación de nuevas empresas y un aumento en la productividad de las ya existentes.

\section{GRUPOS ESTRATÉGICOS EN LAS EMPRESAS DEL SECTOR}

El consenso entre la estrategia corporativa y la de las áreas funcionales de la empresa, tanto desde un punto de vista conceptual como empírico, ha sido objeto de un interés creciente en los últimos años, especialmente por lo que respecta a la vinculación entre las estrategias del sistema de operaciones y la de la empresa (11), (12) y (13). Sobre la base de que el consenso entre estrategias es condición para lograr éxito en el desarrollo de la actividad empresarial, la hipótesis tradicional considera que la estrategia corporativa, decidida en los altos niveles jerárquicos de la organización, condiciona y dirige la actividad de las áreas funcionales de la empresa, incluida, lógicamente, la de operaciones (14). En los trabajos más recientes, sobre todo a partir de la década de los 80, tomó fuerza, sobre todo en el ámbito de las pequeñas y medianas empresas, la consideración de las operaciones como causa y desarrollo de la estrategia corporativa: se trataría de encontrar, en este sistema funcional, la ventaja competitiva que pueda tener la empresa para, sobre esta ventaja, construir la estrategia del negocio (15). La preponderancia en el sector cerámico español de las PYMES y las pautas de desarrollo que han seguido estas empresas podrían confirmar esta hipótesis, sin embargo no se han realizado estudios empíricos hasta la fecha sobre este aspecto.

Uno de los objetivos de este proyecto de investigación es, precisamente, determinar el papel de las operaciones en la estrategia empresarial del sector y para ello incluimos, en la encuesta a las empresas del sector, una serie de cuestiones sobre las prioridades concedidas a los factores que se consideran básicos en la definición de la estrategia empresarial, por una parte, y funcional en el ámbito productivo por otra. También nos hemos interesado por la responsabilidad en las decisiones estratégicas asumidas por los diferentes niveles jerárquicos de la empresa.

Las prioridades concedidas a los factores básicos que permiten alcanzar los objetivos generales de la empresa permiten, en principio, establecer las estrategias empresariales que de forma planificada, o de forma adquirida por la experiencia, han adoptado las empresas (16). Sobre la base de las similitudes en estas prioridades pueden conformarse grupos estratégicos de empresas.

La encuesta ha sido realizada mediante entrevista personal de los autores con gerentes y/o responsables de producción de las empresas. Se ha contactado telefónicamente con el 100\% de las empresas asociadas a ASCER (Asociación Española de Fabricantes de Azulejos y Pavimentos Cerámicos) que representan un $80 \%$ del sector, con el fin de fijar la fecha y hora de la entrevista personal para la realización de la encuesta, obteniéndose una tasa de respuesta del $40 \%$. Finalmente se han realizado encuestas a 81 empresas, 73 de ellas pertenecientes a la provincia de Castellón, 3 a las provincias de Valencia y Alicante y 5 al resto de España. Cabe destacar que algunas empresas cuentan con varios centros de producción. Las empresas encuestadas representan un $40 \%$ de las situadas en la Comunidad Valenciana y un $38 \%$ del total nacional. La encuesta está integrada por 47 preguntas, algunas dedicadas a características generales de la empresa, otras relacionadas con el proceso productivo y otras sobre la estrategia del sistema de operaciones, parte de las cuales se ana- 
lizan en este trabajo. La ficha técnica de la encuesta se encuentra disponible en http: / / www.upv.es/gio.

El análisis de las prioridades concedidas por las empresas encuestadas a los distintos factores que permiten alcanzar los objetivos empresariales establece el siguiente orden para el conjunto de la muestra: 1) la consecución de la máxima eficiencia; 2) el desarrollo de nuevos productos; 3 ) la reducción de los costes de producción; 4) la diferenciación de sus productos en el mercado; 5) la especialización en pocos productos o líneas de productos.

El tamaño empresarial no resulta ser un elemento determinante en la asignación de estas prioridades, al no existir diferencia significativa entre las prioridades concedidas a estos factores por las empresas pequeñas, medianas y grandes.

Para establecer la existencia de grupos estratégicos hemos extraído los factores comunes del conjunto de respuestas sobre prioridades obtenido en la encuesta en relación a los objetivos generales de la empresa. Para la extracción de los factores comunes hemos realizado un análisis factorial, con el que se han extraído tres factores básicos. Se ha utilizado el programa SPSS para la realización del análisis; el método de extracción ha sido el de las componentes principales y aunque la varianza total explicada por los tres factores extraídos no es muy elevada, tanto la medida de adecuación muestral de Kaiser, Meyer y Olkin, como la prueba de esfericidad de Bartlett presentan valores aceptables en este tipo de análisis; también se ha realizado un análisis de fiabilidad para la escala utilizada, obteniéndose un valor de $\alpha$ de 0,6359, superior al límite de aceptación considerado en este tipo de análisis. La rotación efectuada con el método de normalización varimax, nos facilita la asignación de las variables iniciales a los factores, obteniéndose la siguiente agrupación de las cuestiones iniciales:

\section{Factor 1}

Desarrollo de nuevos productos

Diferenciación de sus productos en el mercado

Introducción de nuevos métodos comerciales

Control de los canales de distribución Publicidad

Factor 2

$$
\text { Reducción de costes de producción }
$$
Disponibilidad de materias primas

Factor 3

$$
\text { Minimización de la financiación ajena }
$$

Especialización en pocos productos o líneas de productos Alcanzar la máxima eficiencia

Esta agrupación nos proporcionaría los tres ejes principales de las prioridades competitivas que podemos identificar como estrategia de diferenciación y de costes, en los dos primeros factores, mientras que el tercero agrupa una mezcla de independencia, especialización y eficiencia no muy claramente definida. Las prioridades medias asignadas por las empresas encuestadas a estos factores se han utilizado como elementos para la definición de los grupos estratégicos; para ello se ha empleado el método de agrupación de Ward, utilizando como métrica la distancia euclídea al cuadrado. Se han seleccionado tres grupos con una distribución de 23, 30 y 18 empresas respectivamente (los grupos adicionales serían de tamaño muy reducido y no aportan información más relevante sobre las estrategias empresariales que manifiestan).

Las prioridades medias asignadas por los diferentes grupos a los factores extraídos (tabla 1) permiten establecer los siguientes grupos estratégicos:

Grupo 1: integrado por 23 empresas caracterizadas por dar una prioridad significativamente mayor a la dimensión de diferenciación.
Grupo 2: integrado por 30 empresas con una prioridad significativamente mayor en la dimensión coste.

Grupo 3: integrado por 18 empresas que no presentan diferencias significativas en las prioridades asignadas a los distintos factores pero que en todo caso éstas son muy inferiores a las de los restantes grupos.

Estos grupos son independientes del tamaño empresarial, de hecho la distribución por tamaños de las empresas integradas en los distintos grupos es prácticamente igual a la esperada en base al tamaño muestral, por lo que no podemos asignar un comportamiento estratégico predeterminado a ningún tamaño empresarial.

TABla 1: PRIORIDAdES MEDIAS DE LOS GRUPOS ESTRATÉGICOS (EL VALOR MÁS ALTO DE LA ESCALA ES 5 Y COINCIDE CON LA MAYOR PRIORIDAD)

\begin{tabular}{|l|l|l|l|}
\hline & Diferenciación & Costes & Especialización \\
\hline Grupo 1 & 3.93 & 3.28 & 3.59 \\
\hline Grupo2 & 3.82 & 4.35 & 3.88 \\
\hline Grupo 3 & 3.04 & 3.00 & 2.70 \\
\hline
\end{tabular}

El análisis de estos resultados nos permite pensar, en principio que las empresas encuadradas en los dos primeros grupos disponen de una estrategia definida para alcanzar sus objetivos, mientras que las del tercer grupo no tienen una prioridad estratégica clara, de hecho los valores medios de las tres dimensiones encontradas no difieren significativamente de la posición indiferente de la escala utilizada. Es más, si analizamos las prioridades asignadas a las cuestiones planteadas en la encuesta sobre la forma de alcanzar los objetivos generales de la empresa de forma sistemática, y estadísticamente significativa, se obtienen valores medios inferiores a los asignados por los restantes grupos y, normalmente inferiores al punto de indiferencia de la escala. Solo declaran una prioridad similar a los restantes grupos en la cuestión relativa a los precios competitivos con la competencia, cuestión para la que no existen diferencias significativas entre los grupos competitivos. Es de destacar también que en las empresas del grupo 1 y 2 sólo existen diferencias significativas en las prioridades asignadas en las cuestiones vinculadas al factor costes; en las cuestiones vinculadas al factor diferenciación las medias del grupo 1 son mayores que las del grupo 2 pero no hay diferencias significativas entre ambos grupos. Respecto a los factores condicionantes del entorno competitivo del sector, el poder negociador de los clientes es el considerado más importante por el conjunto de las empresas encuestadas (tabla 2), sin embargo, la rivalidad entre competidores es percibida por todas las empresas con similar importancia Este es el único factor en el que las respuestas obtenidas no indican la existencia de diferencias significativas entre los grupos competitivos estudiados. El factor al que se le asigna menor importancia para la definición del entorno competitivo es el de existencia de productos sustitutivos. En todos los casos son las empresas integradas en el grupo 2 las que asignan más importancia a todos los factores.

TABLA 2: IMPORTANCIA CONCEDIDA POR LAS EMPRESAS A LOS FACTORES DEL ENTORNO COMPETITIVO (EL VALOR MÁS ALTO DE LA ESCALA ES 5 Y COINCIDE CON LA MAYOR PRIORIDAD)

\begin{tabular}{|l|c|c|c|c|}
\hline Factor & $\begin{array}{c}\text { Grupo } \\
1\end{array}$ & $\begin{array}{c}\text { Grupo } \\
2\end{array}$ & $\begin{array}{c}\text { Grupo } \\
3\end{array}$ & $\begin{array}{c}\text { Media } \\
\text { general }\end{array}$ \\
\hline Entrada de nuevos competidores & 2.96 & 3.53 & 2.67 & 3.13 \\
\hline Aparición de productos sustitutivos & 2.87 & 3.13 & 1.89 & 2.73 \\
\hline Rivalidad entre competidores & 3.70 & 3.67 & 3.56 & 3.65 \\
\hline Poder negociados de proveedores & 3.57 & 3.97 & 2.61 & 3.49 \\
\hline Poder negociador de clientes & 4.00 & 4.13 & 3.11 & 3.83 \\
\hline
\end{tabular}


La introducción de nuevos productos aparece como el elemento de más rápido cambio al que se enfrentan las empresas del sector, seguida de los cambios en los gustos y preferencias de los consumidores. Los cambios en los aspectos tecnológicos del proceso productivo no se consideran tan rápidos. Como puede apreciarse en la tabla 3, las empresas del grupo 1 perciben una vida más corta de los productos (más rapidez en la obsolescencia de los productos actuales, en la introducción de nuevos productos y cambios en las preferencias de los consumidores), mientras que las del grupo 2 consideran más rápidos los cambios en el entorno tecnológico. No obstante las diferencias significativas provienen de la baja percepción en la velocidad de cambio de estos factores que manifiestan las empresas del grupo 3.

TABLA 3: VELOCIDAD DE CAMBIO PERCIBIDA POR LAS EMPRESAS (EL VALOR MÁS ALTO DE LA ESCALA ES 5 Y COINCIDE CON LA MAYOR PRIORIDAD)

\begin{tabular}{|l|c|c|c|c|}
\hline Factor & $\begin{array}{c}\text { Grupo } \\
1\end{array}$ & $\begin{array}{c}\text { Grupo } \\
2\end{array}$ & $\begin{array}{c}\text { Grupo } \\
3\end{array}$ & $\begin{array}{c}\text { Media } \\
\text { general }\end{array}$ \\
\hline Obsolescencia de los productos & 3.48 & 3.33 & 3.11 & 3.32 \\
\hline Introducción de nuevos productos & 4.13 & 4.10 & 3.33 & 3.92 \\
\hline Introducción de nuevos procesos & 3.13 & 3.20 & 2.56 & 3.01 \\
\hline Introducción de nuevas tecnologías & 3.30 & 3.47 & 2.39 & 3.14 \\
\hline Preferencias de consumidores & 3.87 & 3.73 & 3.44 & 3.70 \\
\hline
\end{tabular}

La prioridad concedida a los aspectos relativos al cumplimiento de los objetivos básicos del sistema de operaciones de las empresas encuestadas (coste, servicio, calidad y flexibilidad), presentan, en general, valores más altos y menos dispersos que los relativos a los objetivos generales que hemos mencionado anteriormente. Las prioridades medias más altas se obtienen para los aspectos vinculados al objetivo de servicio, seguido de el de costes y flexibilidad, mientras que los niveles más bajos se observan en los aspectos relativos al objetivo de calidad (tabla 4). A nivel de grupos estratégicos, los valores medios obtenidos siguen la pauta esperada del comportamiento de los grupos descrito. Las empresas del grupo 1 presentan niveles de prioridad más altos en los aspectos relativos al servicio (rapidez en la entrega de pedidos y cumplimiento de plazos), mientras que las del grupo 3 presentan los mayores valores en el resto de las cuestiones y muy especialmente en las relativas al control de costes de producción; no obstante, las diferencias manifestadas en la prioridad media entre estos grupos no son significativas, salvo en el caso de los costes; también en estos aspectos son las empresas del grupo 2 las que manifiestan, de forma sistemática y significativa niveles de prioridad más bajos en todos los aspectos. En las cuestiones relativas a la responsabilidad en la toma de decisiones no se han observado diferencias significativas entre los grupos, destacando, en todos los casos planteados la escasa importancia que a la asesoría externa a la empresa se concede como soporte para la toma de decisiones.

\section{CONCLUSIONES}

El estudio realizado nos ha permitido clasificar las empresas del sector en tres grupos que podrían asimilarse a grupos estratégicos. Estos grupos son independientes del tamaño empresarial y se establecen en base a las prioridades concedidas a los factores que definen la forma de alcanzar tanto los objetivos generales de las empresas como los específicos del área de producción, observándose una correspondencia entre los mismos dentro de cada grupo establecido. No obstante, no hemos encontrado una relación directa entre los grupos establecidos y comportamientos distintos en la toma de decisiones y sus responsables en las dis-
TABLA 4: PRIORIDADES CONCEDIDAS AL CUMPLIMIENTO DE LOS OBJETIVOS EN EL ÁREA DE PRODUCCIÓN (EL VALOR MÁS ALTO DE LA ESCALA ES 5 Y COINCIDE CON LA MAYOR PRIORIDAD)

\begin{tabular}{|l|c|c|c|c|}
\hline Factor & $\begin{array}{c}\text { Grupo } \\
1\end{array}$ & $\begin{array}{c}\text { Grupo } \\
2\end{array}$ & $\begin{array}{c}\text { Grupo } \\
3\end{array}$ & $\begin{array}{c}\text { Media } \\
\text { general }\end{array}$ \\
\hline Reducción de los plazos de entrega & 4.13 & 4.27 & 3.78 & 4.10 \\
\hline $\begin{array}{l}\text { Reducción del tiempo de cambio del } \\
\text { proceso }\end{array}$ & 3.91 & 4.07 & 3.89 & 3.97 \\
\hline $\begin{array}{l}\text { Capacidad para introducir cambios } \\
\text { en la programación }\end{array}$ & 3.74 & 4.27 & 3.78 & 3.97 \\
\hline Control estadístico de calidad & 3.83 & 4.27 & 3.06 & 3.82 \\
\hline Control del proceso en tiempo real & 3.83 & 4.10 & 2.50 & 3.61 \\
\hline $\begin{array}{l}\text { Desarrollo de nuevos procesos de } \\
\text { control }\end{array}$ & 3.43 & 3.97 & 2.50 & 3.42 \\
\hline $\begin{array}{l}\text { Incorporar últimas innovaciones } \\
\text { técnicas en los equipos }\end{array}$ & 3.70 & 3.93 & 3.00 & 3.62 \\
\hline Rapidez en la entrega de pedidos & 4.30 & 4.30 & 3.78 & 4.17 \\
\hline Cumplir plazos de entrega & 4.57 & 4.43 & 4.11 & 4.39 \\
\hline Reducir existencias & 3.91 & 3.77 & 2.83 & 3.58 \\
\hline $\begin{array}{l}\text { Incrementar utilización de } \\
\text { instalaciones }\end{array}$ & 3.70 & 4.10 & 3.39 & 3.79 \\
\hline Reducir los costes de producción & 4.09 & 4.63 & 3.67 & 4.21 \\
\hline
\end{tabular}

tintas áreas funcionales de las empresas encuestadas. La similitud de las estructuras organizativas de las empresas del sector podría explicar esta ausencia de diferencias, también es cierto que uno de los grupos obtenidos, el 3, podemos caracterizarlo más por la ausencia de una prioridad competitiva que por la presencia de la misma y que las empresas encuadradas en los otros pueden encontrarse en fase de definición o establecimiento de las mismas ante los recientes cambios operados en el entorno empresarial y, por lo tanto, el establecimiento de prioridades competitivas no ha condicionado aún los procesos de toma de decisiones.

\section{BIBLIOGRAFÍA}

1. E- Criado, M. Regueiro y E. Sánchez . "La industria cerámica en España (1990-2000)” Bol. Soc Esp. Ceram. V. 40 [6] 413-428. 2001

2. E. Criado , M. Regueiro y E. Sánchez. "La industria cerámica española, zante un cambio de ciclo? “Bol. Soc. Esp. Ceram. V. 43 [1] 85-101. 2004

3. J.H. Lingle y W.A. Schiemann. "From balanced scorecard to IS management". Management Review, 56-61. 1996

4. M.E. Porter. "Clusters and competition: new agendas for companies, governments and institutions". Harvard Business School Press. Mass. 1998

5. B. Nooteboom. "Institutions and forms of coordination in innovation systems". Organization Studies vol. $21 \mathrm{n}^{\circ}$ 95: 9-15. 2000

6. C. Camisón y J. Molina. “ El distrito industrial cerámico valenciano:¿mito o realidad competitiva?". Revista Valenciana d'Etudis Autonomics, nº 22 83-102. 1998

7. J. Albors y J. Molina . "La difusión de la innovación, factor competitivo en las redes interorganizativas. El caso de la cerámica valenciana". Revista de Economía Industrial n 339 167-175. 2001

8. R. Cervelló y E. De la Poza. "Impacto económico financiero de las medidas correctoras medioambientales en la industria azulejera de Castellón". Trabajo fin de carrera. Facultad de Administración y Dirección de Empresas, Universidad Politécnica de Valencia. 2003

9. A. Rialp. Fundamentos teóricos de la organización de empresas, Ed. Pirámide. Madrid. 2003

10. A. Escardino. "La innovación tecnológica en la industria cerámica de Castellón”. Bol. Soc. Esp. Ceram.V. 40 [1] $43-51.2001$

11. P. T. Ward y R. Duray. "Manufacturing Strategy in context: environment, competitive strategy and manufacturing strategy". Journal of Operations Management 18, 123-138. 2000

12. S. Chang, C. Yang, H. Cheng y C. Sheu. "Manufacturing flexibility and business strategy: an empirical study of small and medium sized firms". International Journal of Production Economics, 83, 13-26. 2003

13. M. P. Joshi., R. Kathuaria. y S. J. Porth. "Alignment of strategic priorities and perfomance an integration of operations and strategic management perspectives". Journal of Operations Management 21, 353-369. 2003

14. K. Demeter. "Manufacturing strategy and competitiveness". International Journal of Production Economics, 81-82, 205-213. 2003

15. D. Kathuria. "Competitive priorities and managerial perfomance: a taxonomy of small manufacturers". Journal of Operations Management 18, 627-641. 2000

16. F.J. Sáez. "El análisis competitivo en el proceso de formulación de la estrategia empresarial". Revista de Economía y empresa. 33 Vol XII (2ª epoca), 11-31. 1998 